\title{
Research Paper: The Reliability and Concurrent Validity of Digital Inclinometer, Smartphone Applications, and the Cervical Range of Motion Device for Measuring the Cervical Range of Motion
}

\author{
Faezeh Ghorbani $^{1}$ (D), Mojtaba Kamyab ${ }^{1 *}$ (D), Fatemeh Azadinia' ${ }^{1}$ (D), Amir Ahmadi ${ }^{\text {(D) }}$
}

1. Department of Orthotics and Prosthetics, School of Rehabilitation Sciences, Iran University of Medical Sciences, Tehran, Iran. 2. Department of Physical Therapy, School of Rehabilitation Sciences, Iran University of Medical Sciences, Tehran, Iran.

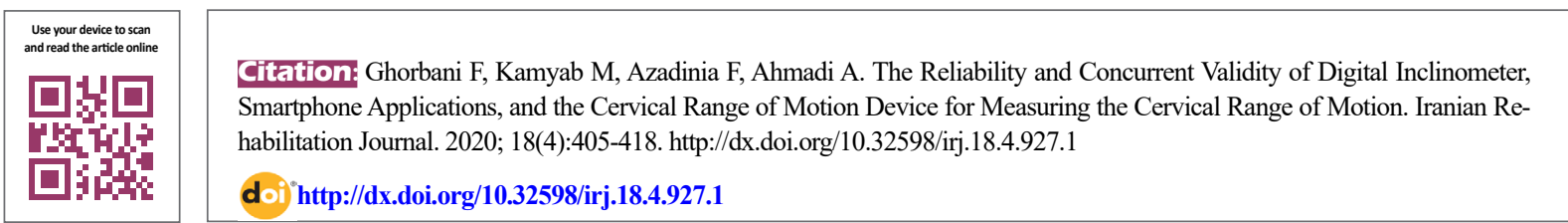

Article info:

Received: 19 Aug 2019

Accepted: 23 Nov 2020

Available Online: 01 Dec 2020

Keywords:

Spine, Cervical vertebrae,

Movement, Phone,

Reproducibility of results

\begin{abstract}
A B S T RACT
Objectives: Changes in the Range of Motion (ROM) are essential criteria in determining the severity of spinal disorders and could be effective in predicting pain progression. Instruments to measure the ROM are costly and unavailable in most therapy settings. While there is a tendency in therapists to use their smartphones instead, there is no report to measure the suitability of smartphones to be employed for this purpose. The current study aimed to compare the interand intra-rater reliability and concurrent validity of a Cervical Range of Motion (CROM) device (as a gold standard), a digital inclinometer, and smartphones in measuring the CROM in asymptomatic adults.
\end{abstract}

Methods: Twenty-four healthy subjects (11 women, 13 men) aged 22 to 45 years were recruited for this study. Neck movements were assessed per study subject using CROM device, dual digital inclinometer, as well as IOS (for iPhone), and Android applications. Despite the popularity of using smartphone applications, there was no study comparing such applications.

Results: The dual inclinometer and iPhone clinometer and compass applications presented acceptable absolute and relative reliability $(\mathrm{ICC}=0.662-0.913)$ and $(\mathrm{ICC}=0.753-0.887$ ), respectively for neck movements in all planes. The reliability of the Android clinometer application in the sagittal and frontal planes was also acceptable (ICC $=0.76-0.937)$; however, the Android compass application used in the horizontal plane indicated the least intraclass correlation coefficient (ICC: $0.202-0.433$ ) in this area.

Discussion: All the tested tools differed from the gold standard depending on the direction of movement, confirmed i.e. approved by the Bland-Altman. The dual digital inclinometer presented moderate to high agreement to the CROM device for all motions, except for rotation. The iPhone applications had high to a very high agreement, and the Android application revealed poor to a moderate agreement. These discrepancies should be considered in employing smartphones for diagnosing a cervical disorder and determining a therapeutic plan. However, as phone applications indicated desirable reproducibility, these tools could be used for the follow-up and monitoring of changes in the CROM. 


\section{Highlights}

- The dual inclinometer revealed acceptable reliability, compared to the gold standard instrument for measuring the cervical range of motion.

- The iPhone clinometer and compass applications could be used as reliable and available tools for assessing the cervical range of motion.

- The relative and absolute reliability of the Android clinometer application was acceptable for use in the sagittal and frontal planes.

- Android compass application illustrated poor reliability in the horizontal plane.

\section{Plain Language Summary}

The reduction of the Cervical Range of Motion (CROM) is among the functional deficiencies induced by neck pain and could greatly affect a person's ability to perform daily living activities. ROM is considered a useful diagnostic indicator, determining the severity of cervical spine impairment and functional limitation. Based on this study, therapists should consider the accuracy and differences of the clinometer and the compass applications of smartphones when using them for assessing the CROM.

\section{Introduction}

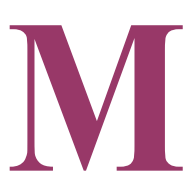

easuring The Range of Motion (ROM) is an integral part of the physical and functional evaluation of patients with musculoskeletal disorders [1, 2]. Accurate ROM evaluations of the cervical spine are challenging due to multiple joint axes and movement coupling [3]. This likely explains why various techniques, ranging from inclinometer to radiographic evaluation and three-dimensional motion analyses, have been used to measure the Cervical Range of Motion (CROM) in clinical practice and scientific studies $[4,5]$. Despite high accuracy, some of these techniques are difficult to be implemented in routine rehabilitation practice. This is because their use is associated with more expenses and time. Most studies suggest employing the CROM device, universal goniometer, and digital inclinometer; however, in practice, some clinicians prefer smartphones to either one of the mentioned expensive instruments or just due to being satisfied with visual estimation [6]. Smartphone applications have become increasingly popular in recent years for their convenience, user-friendly design, and cost-effectiveness. However, a systematic review failed to support smartphone use for evaluating the CROM due to limited studies on smartphone reliability and validity; accordingly, they noted the need for further studies [7]. Most therapists frequently use these applications; thus, it seems necessary to provide information on the extent to which differences would be expected with these instru- ments, compared to clinical diagnostic tools. Accordingly, this study aimed to compare the reproducibility and concurrent validity of clinometer (accelerometer-based) and compass (magnetometer-based), as known applications on IOS (used for iPhone) and Android to those of the CROM device, as the gold standard for non-invasive evaluations [6].

We also investigated the validity and reliability of the dual digital inclinometer here. This is because despite it being the recommended method in the American Medical Association Guide [8], to our knowledge, no study has evaluated its inter- and intra-rater reliability.

\section{Methods}

A convenience sample of Iran University of Medical Sciences students was recruited for this cross-sectional study through advertisements and announcements. Using G-Power software for a minimal significant Intraclass Correlation Coefficient (ICC) value of 0.70 (1$\beta=0.80 ; \alpha=0.05$ ), with a $95 \%$ confidence interval of \pm 0.2 , and an effect size of 0.63 , a minimum of 23 subjects was required. The study participants aged 18-45 years and underwent clinical examinations. Those with a previous head or spinal injury, who underwent surgery for spinal or shoulder girdle disease or pain, or those who reported an intermittent headache within the last 6 months were excluded from the survey [9]. Each study subject provided an informed consent form to participate in the study. 
The study was approved by the Ethics Committee of Iran University of Medical Sciences (no. 27284).

Four non-invasive tools were assessed in the present research, as follows: CROM device (Performance Attainment Associates, Roseville, MN, USA), JTechDualer IQ Digital Inclinometer (JTech Medical, Salt Lake City, UT, USA), Android smartphone (Galaxy A5; Samsung Electronics, Vietnam), and iPhone (6S; Apple Inc, USA). The iPhone $6 \mathrm{~S}$ was equipped with a 6-axis combination gyroscope-accelerometer (InvenSense) and 3-axis accelerometer (Bosch BsMA280). The accuracy and reproducibility of hand-operated tools may be directly influenced by the examiner's expertise; therefore, two orthoses and prostheses doctoral candidates with several years of clinical experience conducted the assessments. An independent observer recorded the data. Before initiating the data gathering process, to warm up and familiarization, after providing instructions on the correct way of performing each cervical motion, the study subjects were allowed to practice the movements for 5 times.

For the CROM assessments in the sagittal and frontal planes, the research subjects sat upright on an 18-in chair with the knees at $90^{\circ}$ of flexion, feet flat on the ground, arms relaxed at the sides, and head and neck in a neutral position [10-12]. The assessment tool selection process was randomized to reduce learning effects, fatigue, and flexibility alterations [13]. Each examiner measured each movement 3 times per tool. Besides, the mean scores of the measurement were used in the statistical analysis. To determine intra-rater reliability, the test-retest sessions were conducted 7 days apart [5] by the lead researcher (F. Gh). All test-retest conditions, including time, place, tools, and inter-trial rest interval were similar. Both examiners independently evaluated the study participants and recorded their data. Additionally, the examiners were blinded to each other's measurements.

For the sagittal assessments, the examiner secured the CROM device to the study participant's head (Figure 1). To assess cervical movements in the sagittal plane, a digital inclinometer was employed. When measuring the smartphone's clinometer application, the examiner snugly held the smartphone in both hands (Figure 2). The examiner stood beside the research subject, who was instructed to flex the neck to the point of tightness or discomfort; return it to a neutral position by staring at the spot on the wall; then extend it as much as possible.

Frontal motion assessments by the CROM device were performed with the examiner in front of the study participant reading the values on the forehead. For the digital inclinometer, a primary sensor was attached to a head strap at the back of the head. Moreover, a secondary sensor was similarly held by the examiner on the scapular spine (Figure 3). The smartphone was held at the back of the study participant's head to measure the frontal plane motions using the clinometer application. After calibration, the research subject was instructed to start in a neutral position; laterally flex the neck to the right maximally; return to the neutral position, and laterally flex the neck to the maximum left.

The rotation arm of the CROM device was added to the tool. Next, the magnetic yoke was placed on the study subject's shoulder for calibration. The examiner was standing directly behind and above the research subject and could see the subject's top of head and tip of the nose. The examiner then stated: "when staring at an imaginary horizontal line on the wall, turn your head to the point of tightness or discomfort". To avoid thoracic and shoulder girdle movements, the study subject was also instructed as follows: "do not move your shoulders or change the extent of pressure being applied to the backrest of your chair".

Cervical ROM assessments were conducted according to the digital inclinometer's catalog using only the primary sensor. The rotation was measured with the study subjects in the supine position on a bench; the examiner was also sitting on a chair holding the primary sensor on the research subject's forehead. A 10-min rest was allowed between conditions. For the horizontal motion assessment using the smartphone compass application, the examiner stood behind the study participant. Accordingly, the examiner adjusted the neutral position and snugly secured the phone on the study subject's vertex using the fingers and palms (Figure 4). After reading the initial degree, the examiner instructed the research subject to rotate to the right and pause for a second, when the assistant read the appearing degree. The study participant then returned to the neutral position, the assistant read the degree, and the participant rotated to the left.

Data normality was investigated using the ShapiroWilk test. ICC models 3, k, and 2, k were used for the inter-and intra-rater analyses, respectively. Cicchetti's [14] classification was used to interpret the relative reliability indices. Accordingly, reliability of ICC $<0.40$ was poor, 0.40-0.59 was moderate, 0.60-0.74 was good, and 0.751 was excellent. The Standard Error of Measurement $(\mathrm{SEM}=\mathrm{SD} \times \sqrt{ }(1-\mathrm{ICC}))$ was calculated to evaluate absolute reliability. This is an estimate of the error value associated with the measurement, i.e. used to calculate the Minimal Detectable Change $(\mathrm{MDC}=\sqrt{ } 2 \times 1.96 \times \mathrm{SEM})$. 


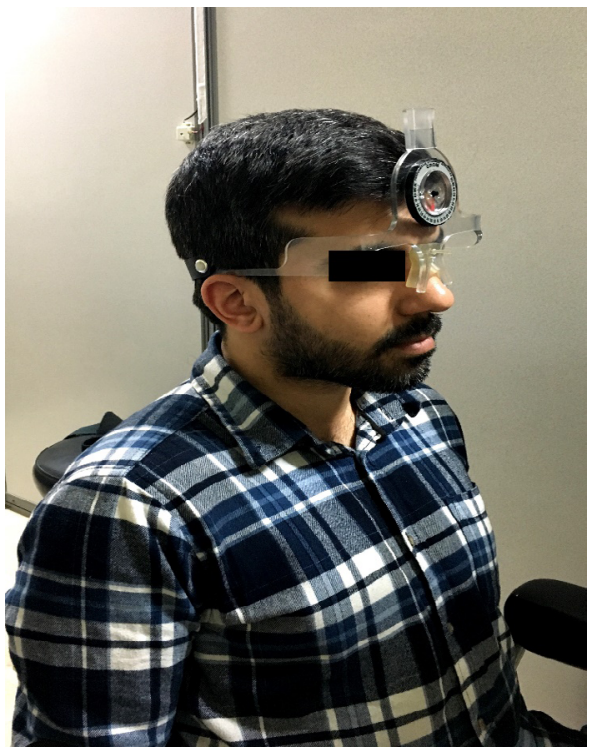

Iranian Rehabilitation Journa

Figure 1. The measurement of cervical rotation with CROM device, while the subject sat on the chair in a straight position

This measure indicates a clinically-significant intermeasurement difference. The Pearson correlation coefficient was calculated to verify the validity of the iPhone, Android smartphone, and dual digital inclinometer. The same test was applied to determine the correlations between their measured ROM and that of the CROM device. The correlation size with the rule of thumb suggest-

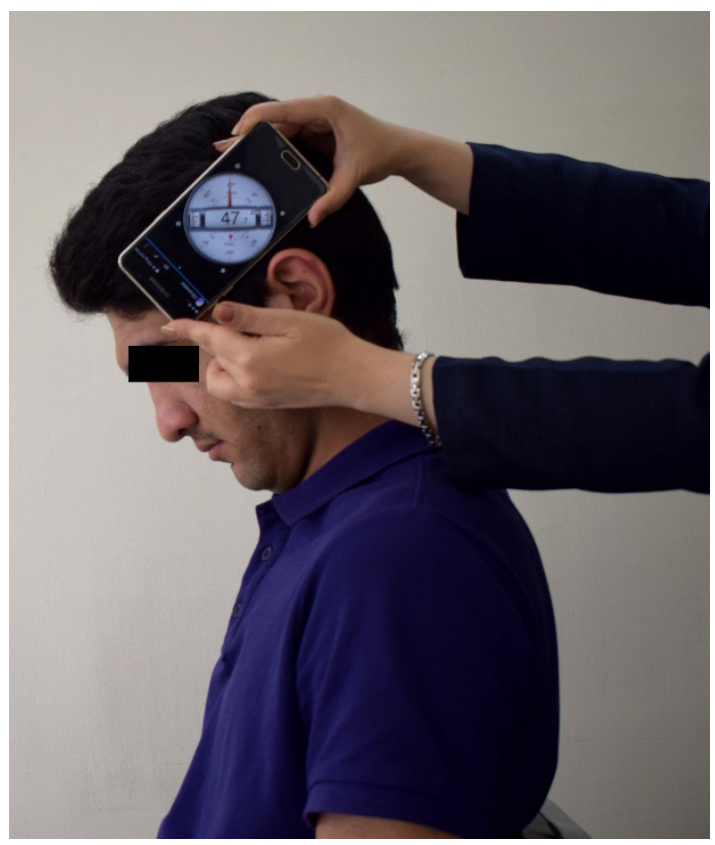

Iranian Rehabilitation Journa Figure 3. The CROM measurement in the fontal plane using a dual digital inclinometer

Two sensors were utilized to assess lateral flexion range of motion in 3 trials as the subject was sitting on the chair.

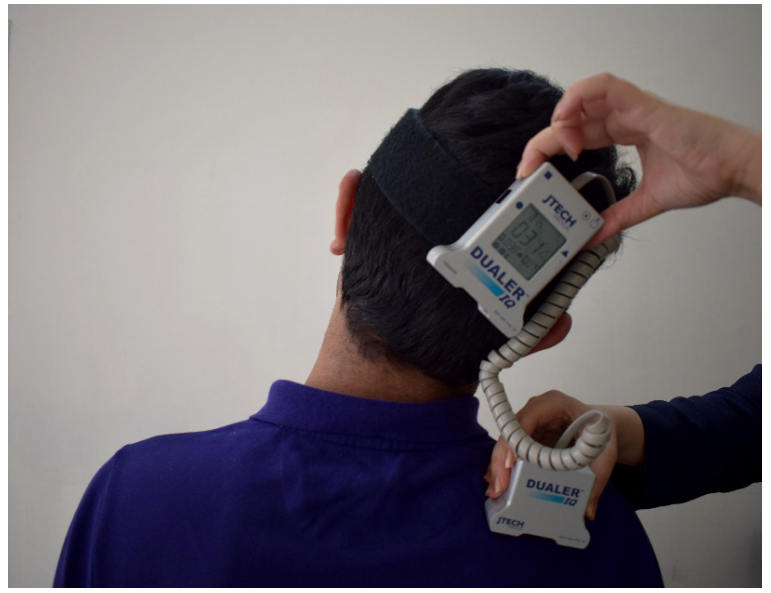

Iranian Rehabilitation Journal

Figure 2. Assessing the CROMs in sagittal plane by Android clinometer application

The examiner placed the smartphone and asked the subject to flex and extend.

ed by Mukaka was interpreted as negligible (0.00-0.30), low (0.30-0.50), moderate (0.50-0.70), high (0.70-0.90), or very high (0.90-1). A Bland-Altman plot was drawn to determine the agreement in ROM measured with the iPhone, the Android smartphone, and the dual digital inclinometer to the ROM measured using the CROM device. The Bland-Altman plot was plotted per tool from the average mean measurements obtained by a tool and the gold standard versus their differences (Appendix A, B \& C). The 95\% limits of agreement were calculated using the equation Limits of Agreement (LOA) $=95 \%$ Mean Difference \pm 1.96 SD, which specifies the limit of difference between the two tools for $95 \%$ of the time.

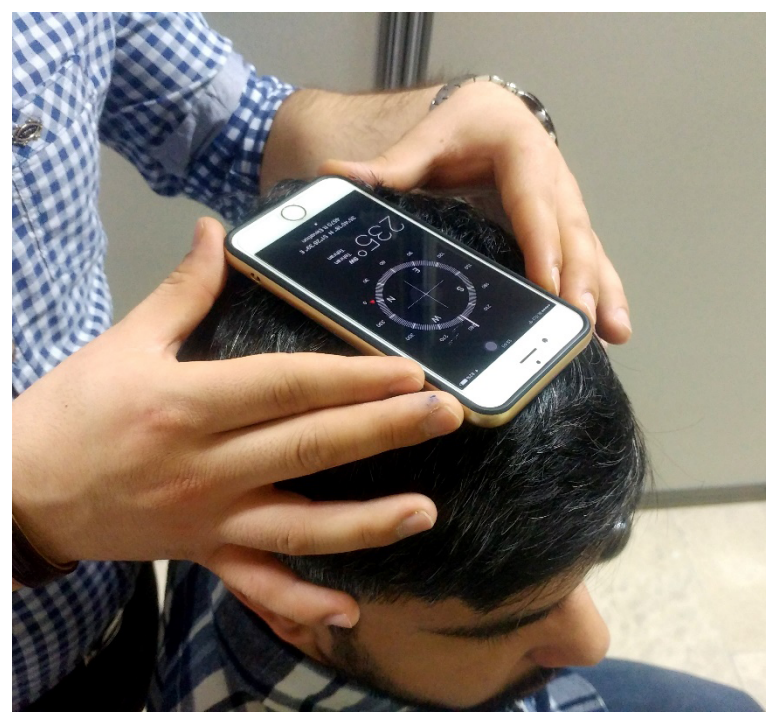

Iranian Rehabilitation Journal Figure 4. Assessing the horizontal plane of cervical range of motion by smartphone compass application 
The main investigator (F.Gh) rated intra-rater reliability and criterion validity.

\section{Results}

Twenty-four volunteers (11 women, 13 men) with the Mean \pm SD age of $28.78 \pm 1.15$ years (range: $22-40$ years) participated in this study. Table 1 displays the study subjects' demographic details. One research participant failed to attend the second evaluation session; therefore, the intra-rater results include 23 cases. The Mean $\pm \mathrm{SD}$ scores of the ROM measurements for all devices and directions are provided in Table 2.

The CROM device and digital inclinometer presented excellent reliability for all planes (ICC $=0.76-0.92 \&$ 0.76-0.91, respectively) and desirable inclinometer reliability for flexion (ICC=0.62). The iPhone's clinometer and compass applications indicated excellent intra-rater reliability for measuring cervical motion ( $\mathrm{ICC}=0.75$ $0.88)$. In cervical motion assessments, the intra-rater reliability of the Android's smartphone clinometer and compass in the sagittal and frontal planes revealed excellent inter-rater reliability ( $\mathrm{ICC}=0.76-0.93$ ); whereas that of the compass application was moderate for the left rotation $(\mathrm{ICC}=0.43$ ) and poor for the right rotation $(\mathrm{ICC}=0.20)$ (Table 3).

For the CROM device assessments, the inter-rater reliabilities in the sagittal, frontal, and horizontal planes were measured as excellent ( $\mathrm{ICC}=0.84-0.96)$. The digital inclinometer demonstrated excellent reliability $(\mathrm{ICC}=0.75$ 0.86 ), except for the right lateral flexion $(\mathrm{ICC}=0.74)$ and left rotation $(\mathrm{ICC}=0.72$ ). The inter-rater reliability of the iPhone applications for measuring cervical motion in three planes was excellent $(\mathrm{ICC}=0.79-0.91)$ for all movements, but flexion, i.e. moderate ( $\mathrm{ICC}=0.5)$. The assessments of sagittal and frontal motions using the Android clinometer application were excellent (ICC $=0.88-0.89$ ); however, the reliabilities of the compass application for measuring the right and left rotations were poor $(\mathrm{ICC}=$ $0.01 \& \mathrm{ICC}=0.21$, respectively) (Table 4 ).

The validity analysis of the dual digital inclinometer indicated a high correlation between extension and right lateral flexion; a moderate correlation between left lateral flexion, flexion, and right rotation; as well as a low correlation for left rotation (Table 5). The iPhone presented a high or very high correlation for all movements, except flexion. The validation analysis of the Android smartphone measurements indicated a high correlation for extension and lateral flexion; moderate correlation for flexion; and low correlation for rotation. The BlandAltman plots revealed that the dual digital inclinometer suggested a higher ROM for rotation and lower for other movements, compared to the CROM device. The iPhone illustrated a lower ROM for flexion and extension and a higher ROM for other movements. The Android smartphone tended to reflect greater ROM for right lateral flexion than the CROM device and lower ROM for other motions than the CROM device.

\section{Discussion}

Recently, with the emergence of multiple goniometric applications, smartphones have become popular medical devices among healthcare professionals. Several studies have evaluated the reliability and validity of smartphones for measuring cervical ROM [6, 15-17]. However, no study has compared iPhone and Android smartphone measurements; nor has any study investigated the reliability and validity of the new gyroscope-accelerometer technology of the iPhone 6. Furthermore, despite dual inclinometry being recommended by the American Medical Association [8], no study has verified its validity and reliability. Therefore, the present study aimed to determine the accuracy and inter- and intra-rater reliabilities of the iPhone, Android smartphone, and digital inclinometer. We also intended to determine their correlations with the gold standard (i.e. the CROM device) [6].

Table 1. Demographic data of the study subjects

\begin{tabular}{|c|c|c|c|}
\hline Demographic Variables & Mean $\pm S D$ & Min. & Max. \\
\hline Age (y) & $28.78 \pm 1.15$ & 22 & 45 \\
\hline Height $(\mathrm{cm})$ & $172.25 \pm 7.69$ & 156 & 185 \\
\hline Weight (kg) & $67.66 \pm 8.66$ & 49 & 83 \\
\hline BMI $\left(\mathrm{kg} / \mathrm{m}^{2}\right)$ & $22.84 \pm 0.53$ & 17.18 & 26.03 \\
\hline
\end{tabular}


Table 2. Mean ROM (degree) during the cervical movements

\begin{tabular}{|c|c|c|c|c|c|c|c|c|}
\hline \multirow{3}{*}{ Movements } & \multicolumn{8}{|c|}{ Degree (Mean \pm SD) } \\
\hline & \multicolumn{2}{|c|}{ CROM } & \multicolumn{2}{|c|}{ Inclinometer } & \multicolumn{2}{|c|}{ iPhone } & \multicolumn{2}{|c|}{ Android } \\
\hline & Examiner 1 & Examiner 2 & Examiner 1 & Examiner 2 & Examiner 1 & Examiner 2 & Examiner 1 & Examiner 2 \\
\hline Flexion & $57.15 \pm 7.43$ & $55.31 \pm 9.37$ & $48.15 \pm 8.94$ & $47.5 \pm 6.5$ & $56.81 \pm 9.73$ & $53.77 \pm 5.5$ & $54.01 \pm 9.36$ & $55.88 \pm 9.55$ \\
\hline Extension & $69.5 \pm 11.32$ & $66.48 \pm 10.97$ & $58.73 \pm 11.03$ & $53.45 \pm 12.4$ & $63.08 \pm 9.53$ & $64.18 \pm 10.14$ & $64.43 \pm 11.81$ & $64.41 \pm 10.29$ \\
\hline $\begin{array}{l}\text { Right lateral } \\
\text { flexion }\end{array}$ & $40.45 \pm 8.08$ & $39.36 \pm 8.98$ & $38.41 \pm 7.56$ & $34.02 \pm 6.85$ & $44.19 \pm 8.35$ & $42.83 \pm 7.48$ & $43.45 \pm 8.36$ & $42.72 \pm 7.88$ \\
\hline $\begin{array}{l}\text { Left lateral } \\
\text { flexion }\end{array}$ & $42.62 \pm 6.93$ & $42.51 \pm 7.98$ & $38.18 \pm 9.35$ & $36.87 \pm 7.8$ & $43.01 \pm 6.86$ & $40.26 \pm 6.08$ & $41.75 \pm 6.29$ & $40.61 \pm 6.71$ \\
\hline Right rotation & $68.01 \pm 10.44$ & $67.16 \pm 8.25$ & $74.16 \pm 8.16$ & $74.06 \pm 8.23$ & $74.25 \pm 8.1$ & $76.01 \pm 7.24$ & $64.04 \pm 9.02$ & $70.08 \pm 11.74$ \\
\hline Left rotation & $65.66 \pm 9.24$ & $65.66 \pm 8.57$ & $74.36 \pm 8.03$ & $74.41 \pm 9.94$ & $72.83 \pm 7.16$ & $73.84 \pm 6.4$ & $65.41 \pm 8.76$ & $72.72 \pm 13.12$ \\
\hline
\end{tabular}

Пranian Rehabilitation \ournal

Table 3. Intra-rater reliability, intraclass correlation coefficient, confidence interval ( $95 \% \mathrm{CI})$, standard error of measurement, and minimum detectable chang in the explored devices

\begin{tabular}{|c|c|c|c|c|c|c|c|}
\hline \multicolumn{2}{|c|}{ Movements } & Flexion & Extension & $\begin{array}{l}\text { Right Lateral } \\
\text { Flexion }\end{array}$ & $\begin{array}{l}\text { Left Lateral } \\
\text { Flexion }\end{array}$ & Right Rotation & Left Rotation \\
\hline \multirow{4}{*}{ CROM } & ICC & 0.764 & 0.841 & 0.923 & 0.895 & 0.921 & 0.805 \\
\hline & $95 \% \mathrm{Cl}$ & $0.454-0.904$ & $0.628-0.931$ & $0.819-0.967$ & $0.535-0.946$ & $0.820-0.968$ & $0.54-0.915$ \\
\hline & SEM & 3.972 & 4.759 & 2.243 & 2.413 & 2.871 & 3.682 \\
\hline & MDC & 9.240 & 11.071 & 5.218 & 5.614 & 6.679 & 8.566 \\
\hline \multirow{4}{*}{ Inclinometer } & ICC & 0.662 & 0.913 & 0.85 & 0.812 & 0.765 & 0.825 \\
\hline & $95 \% \mathrm{Cl}$ & $0.118-0.841$ & $0.638-0.957$ & $0.452-0.924$ & $0.565-0.921$ & $0.457-0.903$ & $0.592-0.925$ \\
\hline & SEM & 2.368 & 3.085 & 2.466 & 2.307 & 2.124 & 2.159 \\
\hline & MDC & 5.509 & 7.177 & 5.737 & 5.367 & 4.941 & 5.023 \\
\hline \multirow{4}{*}{ iPhone } & ICC & 0.753 & 0.840 & 0.887 & 0.870 & 0.868 & 0.755 \\
\hline & $95 \% \mathrm{Cl}$ & $0.425-0.895$ & $0.556-0.924$ & $0.705-0.948$ & $0.42-0.933$ & $0.695-0.944$ & $0.404-0.889$ \\
\hline & SEM & 6.147 & 3.928 & 2.398 & 2.281 & 3.435 & 3.388 \\
\hline & MDC & 14.3 & 9.138 & 5.579 & 5.306 & 7.991 & 7.882 \\
\hline \multirow{4}{*}{ Android } & ICC & 0.76 & 0.937 & 0.906 & 0.862 & 0.202 & 0.433 \\
\hline & $95 \% \mathrm{Cl}$ & $0.444-0.9$ & $0.64-0.968$ & $0.773-0.958$ & $0.639-0.936$ & $-0.782-0.648$ & $-0.296-0.748$ \\
\hline & SEM & 3.053 & 3.579 & 2.804 & 2.163 & 10.877 & 7.134 \\
\hline & MDC & 7.103 & 8.326 & 6.523 & 5.032 & 25.304 & 16.596 \\
\hline
\end{tabular}

Iranian Rehabilitation Dournal

ICC: Intraclass Correlation Coefficient; CI: Confidence Interval; SEM: Standard Error of Measurement; MDC: Minimum Detectable Change. 
Table 4. Inter-rater reliability, Intraclass Correlation Coefficient, and Confidence Interval $(95 \% \mathrm{CI})$ in the study tools

\begin{tabular}{|c|c|c|c|c|c|c|c|c|}
\hline \multirow{2}{*}{ Movements } & \multicolumn{2}{|c|}{ CROM } & \multicolumn{2}{|c|}{ Inclinometer } & \multicolumn{2}{|c|}{ iPhone } & \multicolumn{2}{|c|}{ Android } \\
\hline & ICC & $95 \% \mathrm{Cl}$ & ICC & $95 \% \mathrm{Cl}$ & ICC & $95 \% \mathrm{Cl}$ & ICC & $95 \% \mathrm{Cl}$ \\
\hline Flexion & 0.79 & $0.515-0.907$ & 0.793 & $0.532-0.913$ & 0.5 & $-0.131-0.771$ & 0.872 & $0.696-0.942$ \\
\hline Extension & 0.905 & $0.728-0.954$ & 0.86 & $0.477-0.928$ & 0.86 & $0.477-0.928$ & 0.885 & $0.742-0.952$ \\
\hline Right lateral flexion & 0.965 & $0.912-0.984$ & 0.744 & $0.174-0.865$ & 0.919 & $0.803-0.963$ & 0.896 & $0.766-0.956$ \\
\hline Left lateral flexion & 0.905 & $0.79-0.961$ & 0.81 & $0.566-0.918$ & 0.886 & $0.533-0.941$ & 0.889 & $0.739-0.95$ \\
\hline Right rotation & 0.909 & $0.794-0.961$ & 0.757 & $0.449-0.899$ & 0.803 & $0.54-0.911$ & -0.011 & $-0.966-0.525$ \\
\hline Left rotation & 0.84 & $0.639-0.933$ & 0.729 & $0.383-0.887$ & 0.796 & $0.535-0.912$ & 0.218 & $-0.577-0.617$ \\
\hline
\end{tabular}

Iranian Rehabilitation Journal

Table 5. Pearson correlation coefficient data, compared to CROM device data

\begin{tabular}{ccccccc}
\hline \multirow{2}{*}{ Movements } & \multicolumn{2}{c}{ Inclinometer } & \multicolumn{2}{c}{ iPhone } & \multicolumn{2}{c}{ Android } \\
\cline { 2 - 6 } & Pearson, $r$ & $\mathbf{P}$ & Pearson, $\mathbf{r}$ & $\mathbf{P}$ & Pearson, $\mathbf{r}$ & $\mathbf{P}$ \\
\hline Flexion & 0.605 & 0.002 & 0.593 & 0.003 & 0.622 & 0.002 \\
\hline Extension & 0.72 & $<0.001$ & 0.87 & $<0.001$ & 0.73 & $<0.001$ \\
\hline Right lateral flexion & 0.812 & $<0.001$ & 0.909 & $<0.001$ & 0.781 & $<0.001$ \\
\hline Left lateral flexion & 0.616 & 0.002 & 0.917 & $<0.001$ & 0.733 & $<0.001$ \\
\hline Right rotation & 0.675 & $<0.001$ & 0.821 & $<0.001$ & 0.453 & 0.03 \\
\hline Left rotation & 0.313 & 0.143 & 0.817 & $<0.001$ & 0.465 & 0.16 \\
\hline
\end{tabular}

Iranian Rehabilitation Dourna

The present study data demonstrated that the CROM device had excellent inter-day reliability for all cervical motion measurements. Moreover, this is not a new finding and has been supported by numerous studies [4, 11, 12, 18-22]. The dual digital inclinometer also presented excellent inter-day reliability for all cervical motion measurements; the only exception was flexion, which revealed desirable inter-day reliability. Data on the reproducibility of the dual digital inclinometer are scarce. Therefore, it is impossible to compare our results with those of the previous studies. Prushansky et al. [23] investigated the intra-rater reliability of a single digital inclinometer and reported good to excellent reproducibility in all planes. We found excellent intra-rater reliability concerning all planes for the clinometer and compass applications of the iPhone. Tousignant-Laflamme et al. [6] reported moderate to excellent intra-rater reliability for the clinometer application of the iPhone. These data concerned movements in the frontal and sagittal planes. They also found poor to moderate intra-rater reliability for the compass application of the iPhone. Such discrepancy between our data and their results may be attributed to the difference in the number of trials. This is because the number of trials affects the ICC. Consistent with our study findings, Quek et al. [15] reported the intra-rater reliability of the Android smartphone clinometer application to be excellent $(\mathrm{ICC}=0.82-0.9)$. The Android smartphone compass application indicated poor to moderate inter-day reliability in rotation, whereas Quek et al. [15] reported poor intra-rater reliability in this regard $(\mathrm{ICC}=0.05-0.33$ ).

Clinically, absolute reliability indices, such as SEM and MDC are more essential than the relative reliability of measurements. This is because the ICC provides information about the ability to maintain position over repeated measurements [24]. It was unable to identify actual CROM changes that occur as a result of therapeutic interventions. For this reason, SEM and MDC values were extracted from the test-retest paradigm. The patterns of absolute reliability indices were slightly differ- 
ent from those of the relative reliability. Accordingly, the dual digital inclinometer revealed the highest absolute reliability, signifying the lowest SEM and MDC.

The MDC of the iPhone was 5.3 degrees for left lateral flexion to 14.3 degrees for flexion. Concerning the Android smartphone, the lowest MDC was obtained for the left lateral flexion but equaled 16.5 degrees for the left rotation and 25 degrees for the right rotation. These results indicated that a real change in cervical flexion should be equal to $>14.3$ degrees for detection by the iPhone clinometer application. However, a real change in the right and left rotations should be as $>25$ degrees and $>16.5$ degrees, respectively, for detection by the Android compass application. Thus, as the minimum detectable alternations presented high rates, the instrument could not detect the actual changes that occur in a course of treatment. Therefore, the Android smartphone seems to be unable to distinguish individual variations in the horizontal plane. It also fails to detect clinically-relevant changes in re-evaluations during the therapeutic course.

For the CROM device, the inter-rater reliability of the measurements of all motions was obtained as excellent. The inter-rater reliability of the inclinometer and iPhone applications was high, like the intra-rater reliability. Subsequently, consistent results were obtained in measurements repeated by an examiner as were those by a different examiner using the same evaluation procedures. Tousignant-Laflamme et al. [6] reported moderate inter-rater reliability for assessing flexion/extension and lateral/flexion by the iPhone clinometer. There exist possible explanations for such difference between our results and those of Tousignant-Laflamme and associates [6]. In the present study, the tool selection order was randomized to reduce the variability caused by fatigue, learning effect, and flexibility alteration; however, measurement tool use order was the same in the study of Tousignant -Laflamme et al. [6] and iPhone measurements were always performed before CROM device assessments. For rotation, the iPhone compass application revealed poor inter-rater reliability $(\mathrm{ICC}=0.07-0.09)$. Moreover, the Android clinometer reflected excellent inter-rater reliability values, whereas the Android compass application suggested poor reliability for assessing rotation. Quek et al. [15] performed no interrater reliability evaluations of the Android smartphone.

The correlation coefficients of the inclinometer were calculated as moderate to high, compared to those of the CROM device; the only exception was left rotation, which revealed a low correlation. This is probably because the rotation measurement with the inclinometer was performed in the supine instead of the sitting posi- tion. However, based on the $95 \%$ limit of agreement, the differences in the inclinometer and CROM device varied (2-27 degrees) by movement direction. The iPhone revealed a high to very high correlation with the CROM device for movements, but flexion. However, the differences in the iPhone and CROM device measurements were 1.2-18.6 degrees. The differences between the Android smartphone and CROM device measurements were obtained as 7.8-24.2 degrees. This degree of difference from the gold standard cannot be clinically-overlooked. This is because it affects cervical disorder diagnosis as well as the rating of cervical disorders and therapeutic strategy planning. Another possible justification for interpreting the findings may relate to how we determined neutral head position in the present study. We visually controlled the neutral head position using self-balancing maneuvers. This may have resulted in inconsistent starting positions of all trials and measurement errors with the Android smartphone, iPhone, and dual inclinometer. However, such errors did not occur with the CROM device, because we measured the total range (final minus initial position). In addition, the weaker correlation of the Android smartphone with the CROM device can be attributed to the characteristics of this smartphone, i.e. the Android smartphone calibration likely has low accuracy [25]. Besides, the compass application used to measure horizontal plane motion is highly sensitive to mobile orientation; it is a magnetometer-based application and may easily drift, leading to greater measurement errors.

We acknowledge that this study had a few limitations. The smartphones used in this study were only a few months old; thus, we cannot claim that the same results will be achieved using older smartphones.

\section{Conclusion}

The current research results indicated that the dual inclinometer, iPhone clinometer, and compass applications have acceptable absolute and relative reliability for measuring neck movements in all planes. The reproducibility and accuracy of the Android clinometer application were also acceptable for use in the sagittal and frontal planes; however, its compass application in the horizontal plane revealed the lowest ICC and largest error. According to our results, the assessed tools differed from the gold standard depending on the movement direction; this should be considered when diagnosing cervical disorders and determining therapeutic plans. 


\section{Ethical Considerations}

\section{Compliance with ethical guidelines}

The purpose of the study and the data collection process was explained to the subjects. All subjects participated in this research voluntarily. The study was confirmed by the ethics committee of Iran University of Medical Sciences (no. 27284).

\section{Funding}

This article extracted from a research project and the resource funding was provided by Research Deputy of IUMS at School of Rehabilitation Sciences.

\section{Authors' contributions}

Conceptualization: Mojtaba Kamyab, Fatemeh Azadinia, Faezeh Ghorbani; Methodology, software, validation, formal analysis: All authors; Investigation, visualization: Faezeh Ghorbani, Fatemeh Azadinia; Writing - original draft: Faezeh Ghorbani, Fatemeh Azadinia; Writing - review \& editing: Mojtaba Kamyab, Fatemeh Azadinia; Funding acquisition: Mojtaba Kamyab; Resources: Mojtaba Kamyab, Amir Ahmadi; Supervision: Mojtaba Kamyab.

\section{Conflict of interest}

All authors declared no conflicts of interest.

Acknowledgments

The authors appreciate Shahrbanoo Bidari and Azin Jalali for assisting with the data collection and the study participants.

\section{References}

[1] Kolber MJ, Hanney WJ. The reliability and concurrent validity of shoulder mobility measurements using a digital inclinometer and goniometer: A technical report. International Journal of Sports Physical Therapy. 2012; 7(3):306-13. [PMCID]

[2] Reese NB, Bandy WD. Joint range of motion and muscle length testing. $3^{\text {th }}$ ed. Philadelphia: Saunders; 2016. https:// www.elsevier.com/books/joint-range-of-motion-and-muscle-length-testing/reese/978-1-4557-5882-1

[3] Bogduk N, Mercer S. Biomechanics of the cervical spine. I: Normal kinematics. Clinical Biomechanics. 2000; 15(9):633-48. [DOI:10.1016/S0268-0033(00)00034-6]
[4] Youdas JW, Carey JR, Garrett TR. Reliability of measurements of cervical spine range of motion-comparison of three methods. Physical Therapy. 1991; 71(2):98-104. [DOI:10.1093/ ptj/71.2.98] [PMID]

[5] ordan K, Dziedzic K, Jones PW, Ong BN, Dawes PT. The reliability of the three-dimensional FASTRAK measurement system in measuring cervical spine and shoulder range of motion in healthy subjects. Rheumatology. 2000; 39(4):382-8 [DOI:10.1093/rheumatology/39.4.382] [PMID]

[6] Tousignant-Laflamme Y, Boutin N, Dion AM, Vallée CA. Reliability and criterion validity of two applications of the iPhone $^{\mathrm{TM}}$ to measure cervical range of motion in healthy participants. Journal of Neuroengineering and Rehabilitation. 2013; 10(1):69. [DOI:10.1186/1743-0003-10-69] [PMID] [PMCID]

[7] Rehan Youssef A, Gumaa M. Validity and reliability of smartphone applications for clinical assessment of the neuromusculoskeletal system. Expert review of Medical Devices. 2017; 14(6):481-93. [DOI:10.1080/17434440.2017.1325319] [PMID]

[8] Cocchiarella L, Andersson CBJ. Guides to the evaluation of permanent impairment. $5^{\text {th }}$ ed. Chicago: American Medical Association; 2000. https://www.amazon.com/Guides-Evaluation-Permanent-Impairment-Fifth/dp/1579470858

[9] Evans NR, Hooper G, Edwards R, Whatling G, Sparkes V, Holt $\mathrm{C}$, et al. A 3D motion analysis study comparing the effectiveness of cervical spine orthoses at restricting spinal motion through physiological ranges. European Spine Journal. 2013; 22(1):10-15. [DOI:10.1007/s00586-012-2641-0] [PMID] [PMCID]

[10] Sforza C, Grassi G, Fragnito N, Turci M, Ferrario VF. Three-dimensional analysis of active head and cervical spine range of motion: effect of age in healthy male subjects. Clinical Biomechanics. 2002; 17(8):611-4. [DOI:10.1016/S0268 0033(02)00071-2]

[11] Fletcher JP, Bandy WD. Intrarater reliability of CROM measurement of cervical spine active range of motion in persons with and without neck pain. Journal of Orthopaedic \& Sports Physical Therapy. 2008; 38(10):640-5. [DOI:10.2519/ jospt.2008.2680] [PMID]

[12] Audette I, Dumas JP, Côté JN, De Serres SJ. Validity and between-day reliability of the Cervical Range of Motion (CROM) device. Journal of Orthopaedic \& Sports Physical Therapy. 2010; 40(5):318-23. [DOI:10.2519/jospt.2010.3180] [PMID]

[13] Werner BC, Holzgrefe RE, Griffin JW, Lyons ML, Cosgrove CT, Hart JM, et al. Validation of an innovative method of shoulder range-of-motion measurement using a smartphone clinometer application. Journal of Shoulder and Elbow Surgery. 2014; 23(11):e275-82. [DOI:10.1016/j.jse.2014.02.030] [PMID]

[14] Cicchetti DV. Guidelines, criteria, and rules of thumb for evaluating normed and standardized assessment instruments in psychology. Psychological Assessment. 1994; 6(4):284-90. [DOI:10.1037/1040-3590.6.4.284]

[15] Quek J, Brauer SG, Treleaven J, Pua YH, Mentiplay B, Clark RA. Validity and intra-rater reliability of an Android phone application to measure cervical range-of-motion Journal of Neuroengineering and Rehabilitation. 2014; 11:65. [DOI:10.1186/1743-0003-11-65] [PMID] [PMCID] 
[16] Pourahmadi MR, Bagheri R, Taghipour M, Takamjani IE, Sarrafzadeh J, Mohseni-Bandpei MA. A new iPhone application for measuring active craniocervical range of motion in patients with non-specific neck pain: A reliability and validity study. The Spine Journal. 2018; 18(3):447-57. [DOI:10.1016/j. spinee.2017.08.229] [PMID]

[17] Guidetti L, Placentino U, Baldari C. Reliability and criterion validity of the smartphone inclinometer application to quantify cervical spine mobility. Clinical Spine Surgery. 2017; 30(10):E1359-66. [DOI:10.1097/BSD.00000000000000364] [PMID]

[18] Williams MA, Williamson E, Gates S, Cooke MW. Reproducibility of the cervical range of motion (CROM) device for individuals with sub-acute whiplash associated disorders. European Spine Journal. 2012; 21(5):872-8. [DOI:10.1007/ s00586-011-2096-8] [PMID] [PMCID]

[19] Hickey ER, Rondeau MJ, Corrente JR, Abysalh J, Seymour CJ. Reliability of the cervical range of motion (CROM) device and plumb-line techniques in measuring resting head posture (RHP). Journal of Manual \& Manipulative Therapy. 2000; 8(1):10-7. [DOI:10.1179/106698100790811346]

[20] Rheault W, Albright B, Byers C, Franta M, Johnson A, Skowronek $\mathrm{M}$, et al. Intertester reliability of the cervical range of motion device. Journal of Orthopaedic \& Sports Physical Therapy. 1992; 15(3):147-50. [DOI:10.2519/jospt.1992.15.3.147] [PMID]

[21] Capuano-Pucci D, Rheault W, Aukai J, Bracke M, Day R, Pastrick M. Intratester and intertester reliability of the cervical range of motion device. Archives of Physical Medicine and Rehabilitation. 1991; 72(5):338-40. archives-pmr.org/ article/0003-9993(91)90254-G/pdf

[22] Tousignant M, Smeesters C, Breton AM, Breton É, Corriveau $\mathrm{H}$. Criterion validity study of the cervical range of motion $(\mathrm{CROM})$ device for rotational range of motion on healthy adults. Journal of Orthopaedic \& Sports Physical Therapy. 2006; 36(4):242-8. [DOI:10.2519/jospt.2006.36.4.242] [PMID]

[23] Prushansky T, Dvir Z. Cervical motion testing: Methodology and clinical implications. Journal of Manipulative and Physiological Therapeutics. 2008; 31(7):503-8. [DOI:10.1016/j. jmpt.2008.08.004] [PMID]

[24] Atkinson G, Nevill AM. Statistical methods for assessing measurement error (reliability) in variables relevant to sports medicine. Sports Medicine, 1998; 26(4):217-38. [DOI:10.2165/00007256-199826040-00002] [PMID]

[25] McGhee B. How to calibrate the compass on your Android phone [Internet]. 2018 [Updated 2018 August 4]. Available from: https://www.nextpit.com/how-to-calibrate-compasson-android 
Appendix A. Bland-Altman Plot demonstrating mean differences and 95\% limits of agreement between CROM device and digital inclinometer measurements of the CROM (degrees)

Most values measured by digital inclinometer and CROM device (gold standard) were close to each other with fewer differences.

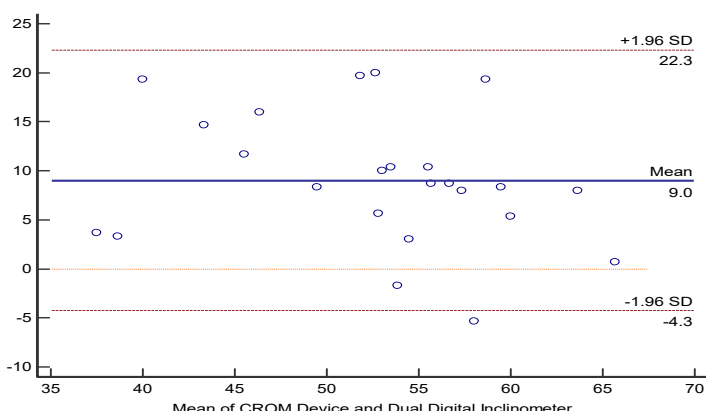

Cervical Right Lateral Flexion

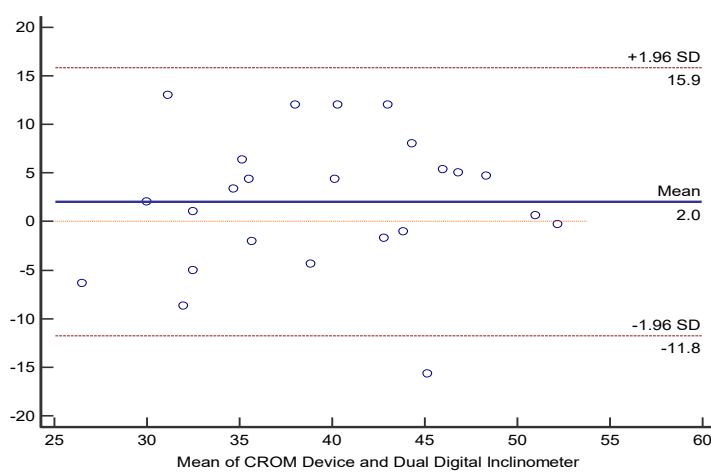

Cervical Right Rotation

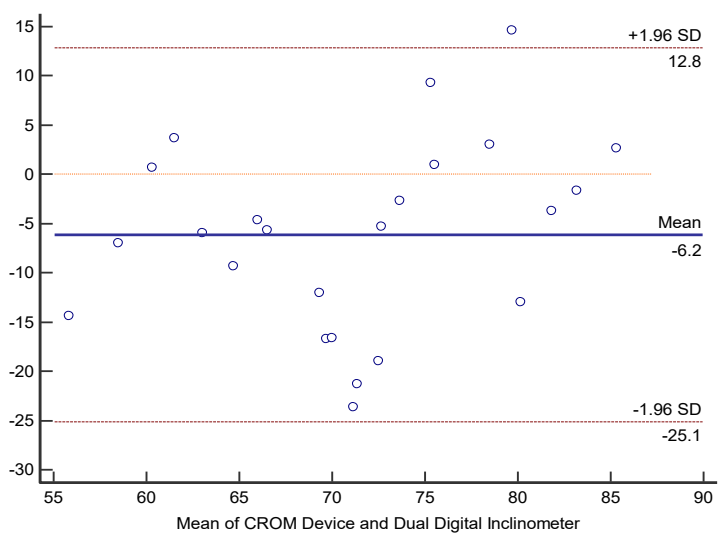

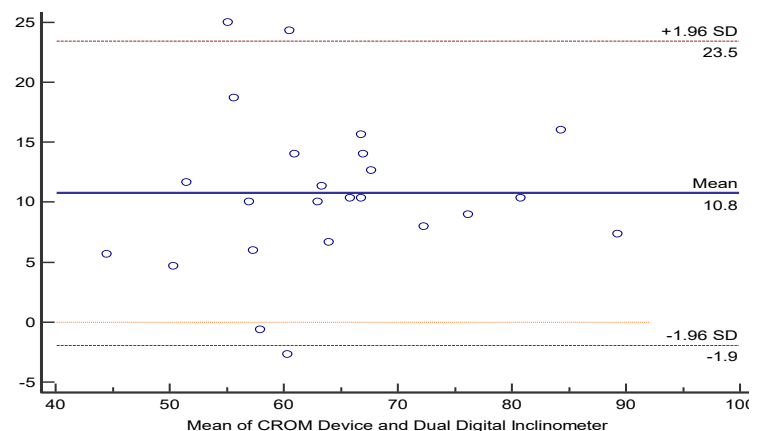

Cervical Left Lateral Flexion

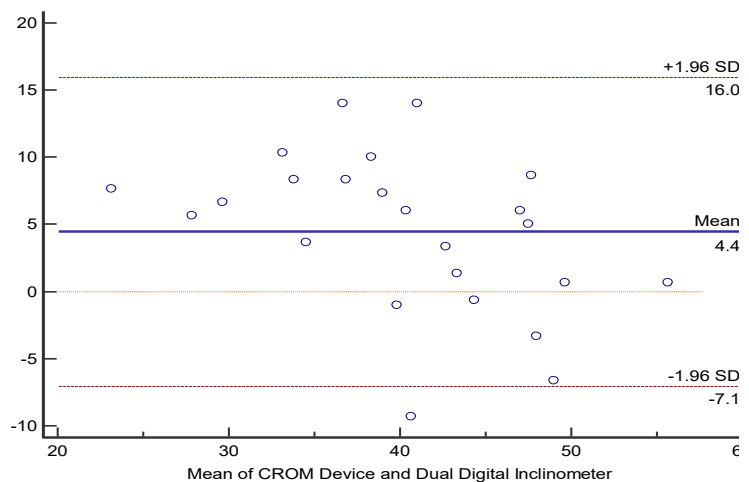

Cervical Left Rotation

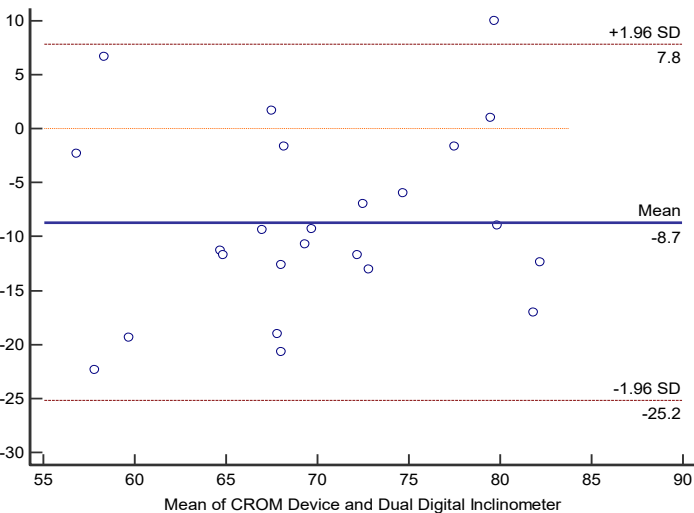

Iranian Rehabilitation \ourna 
Appendix B. iPhone apps measurements of the CROM (degrees)

The visual assessment shown by Bland-Altman Plot, indicate close results for measuring cervical range of motion by CROM device and iPhone apps.

\section{Cervical Flexion}

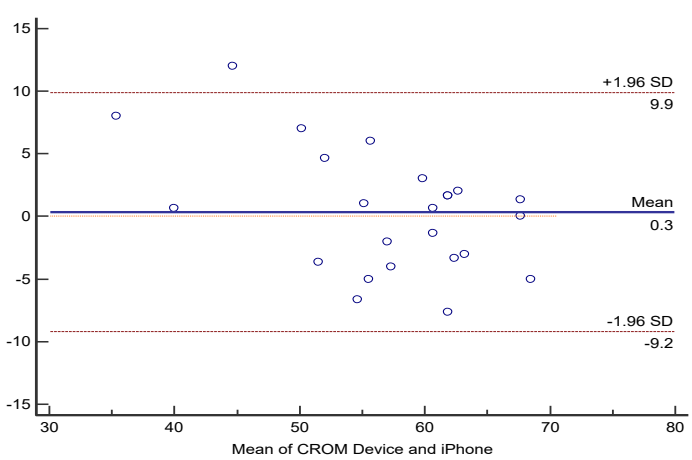

Cervical Right Lateral Flexion

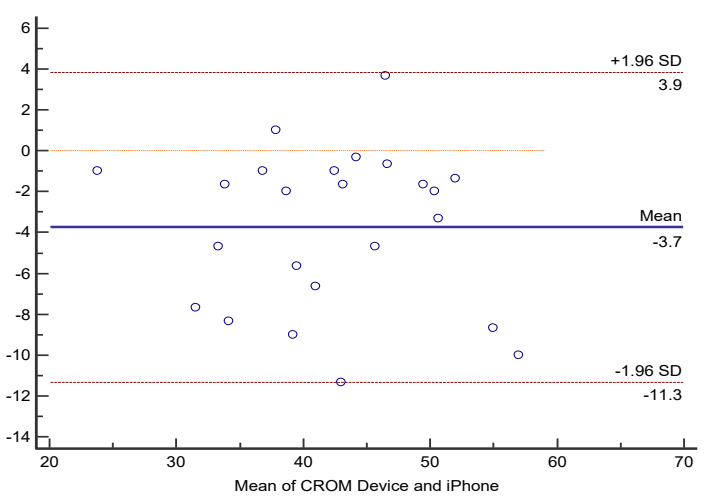

Cervical Right Rotation

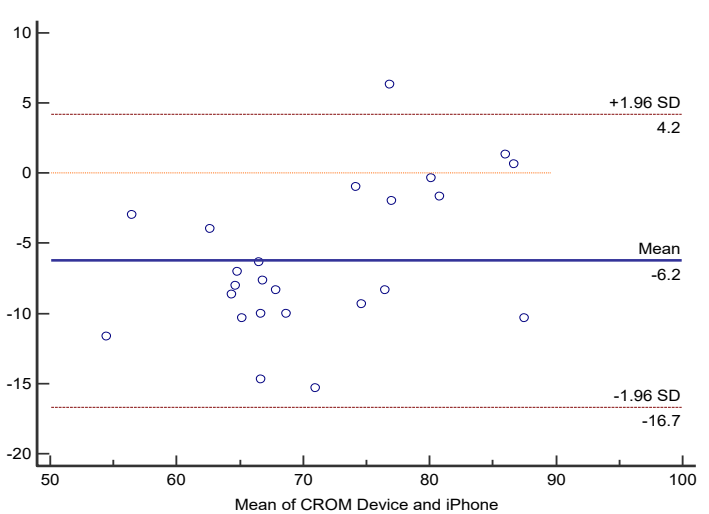

Cervical Extension

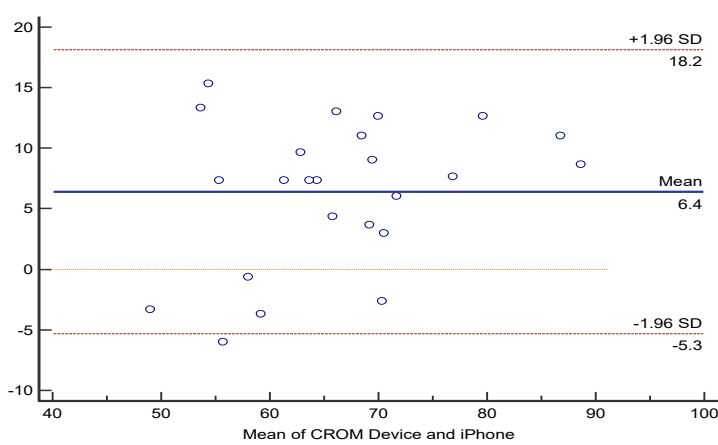

Cervical Left Lateral Flexion

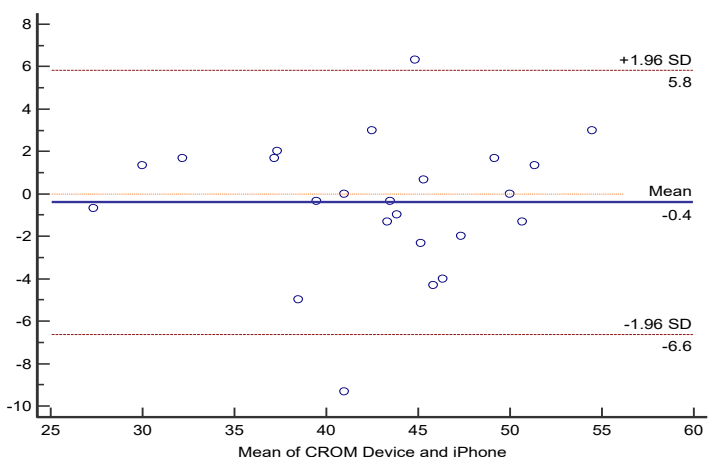

Cervical Left Rotation

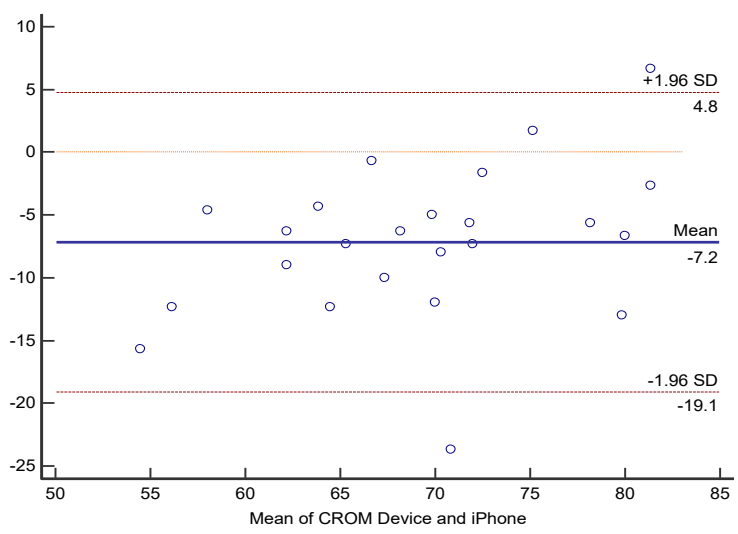

Iranian Rehabilitation Journal 
Appendix C. Bland-Altman Plot showing mean differences and 95\% limits of agreement between CROM device and Android apps measurements of the CROM (degrees)

Based on plots, the cervical range of motion values measured by Android apps shows little difference compare to the CROM device as a gold standard tool.

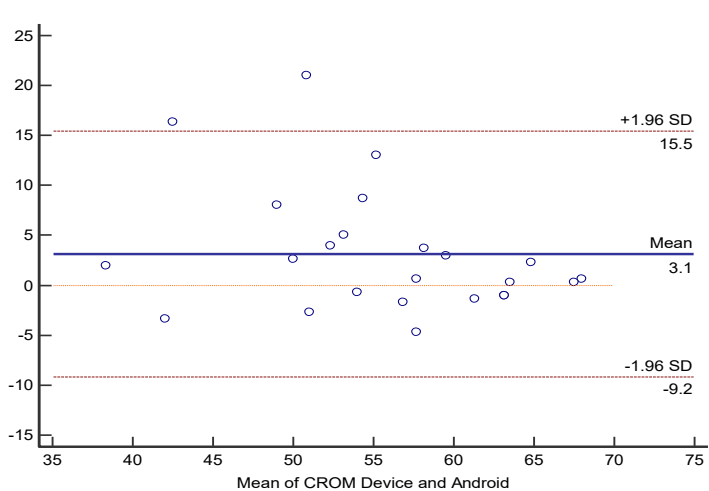

Cervical Right Lateral Flexion

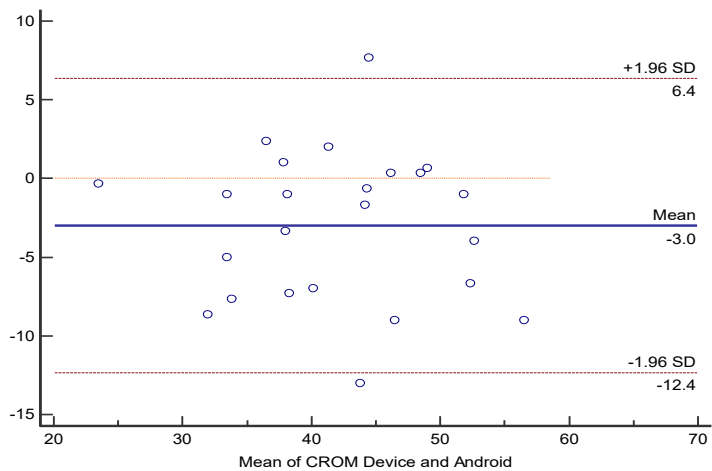

Cervical Right Rotation

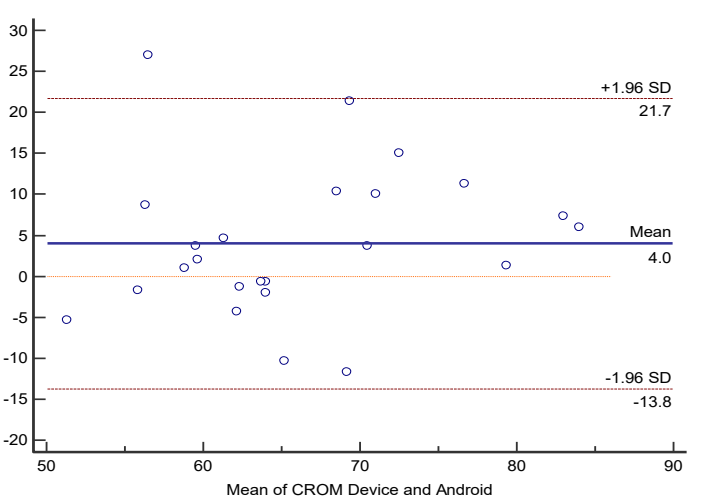

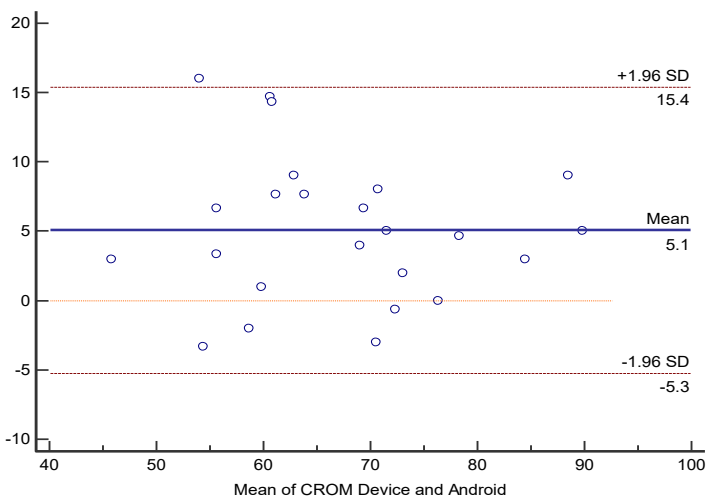

Cervical Left Lateral Flexion

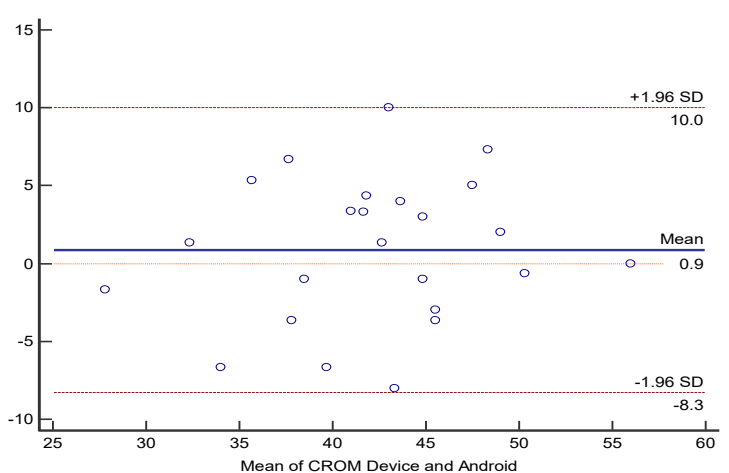

Cervical Left Rotation

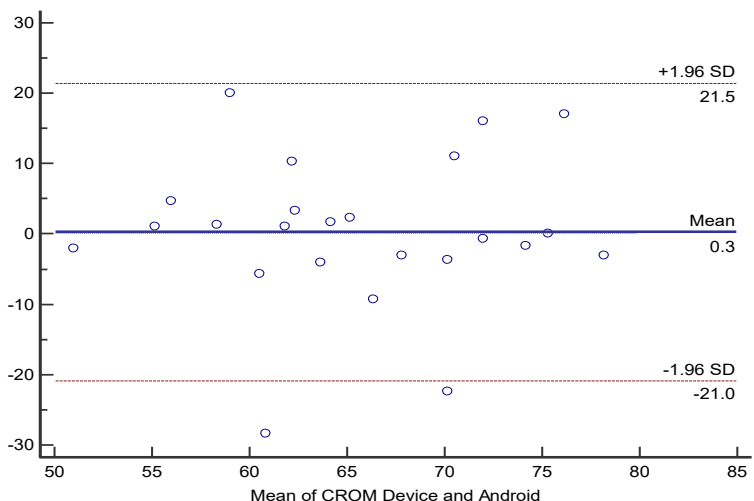

Iranian Rehabilitation Journa 
This Page Intentionally Left Blank 